\title{
Subject Index Vol. 55
}

Alcoholic additives, 41

- $\quad$ cardiomyopathy, 41

Anesthetized dogs, 218

Aortic aneurysms, 351

Aortitis, 351

Arrack, 41

Arrhythmia prognosis, 302 Arteriosclerosis obliterans, 114 Aspartate aminotransf erase isoenzymes,'. ATPase activity, 178

Atrial activation, 69

conduction, 69

ectopic beats, 69

fibrillation, 22, 249, 310, 361

flutter, 310

myxoma, 55

Atrioventricular arrhythmias, $156 \mathrm{~A}-\mathrm{V}$ dissociation, 156

junctional arrhythmias, 156

nodal arrhythmias, 22

/?-adrenergic blockade, 105 y³/8-blockers, 249 Bidirectional tachycardia, 302 Blood flow changes in lower limb, 114

- $\quad$ pressure, 321

Bundle branch block, 242

Calcium binding, 178 Canine transmembrane action potential 340

Cardiac arrhythmias, 65

configuration, 281

measurements, 281

output, 94,105,136 Cardiomyopathy, 84

obscure, 272 Cardiovascular sounds, 129 Cardioversion, 310

Carotid sinus nerve stimulation, 94 Catecholamine cardiomyofibrosis, 84 Claudicating extremities, 114 Closure rate, 237 CNS vasomotor responses, 164 Coarctation of the aorta, 149 Congenital complete heart block, 242

- $\quad$ heart disease, 193

Congestive heart failure, 211

Coronary flow, 193

Cyanosis, 55

Cyanotic heart disease, 193

DC shock, 310 Defibrillation, 310 Diaphragmatic level, 281 Dietetic cardiomyofibrosis, 84

Digital blood flow, 257 Digitalis, 302

- $\quad$ intoxication, 22

Dive reflex, 257 
Ductus arteriosus, 237

Dye dilution technique, 136

Subject Index

375

Electrocardiogram, 149 Electrocardiography exercise, 333 Electronically amplified oscillography,

114 Exercise test, 105

Familial Q waves in electrocardiogram,

272 Fourth heart sound, 333

Glucagon, 211 Guinea pig, 237

Microelectrode, 340 Microfocal lesions, 84 Microphone pickup, 129 Mitochondrial

respiration, 84 Mitral stenosis, 361

valvuloplasty, 34 Myocardial contractility, 321

metabolism, 193

Nodal rhythm, 69 Orienting reflex, 257

Heart, 79

block, 65

failure, 321

innervation, 321

mitochondria, 178

rate, $79,94,257,321$

sarcoplasmic reticulum, 178 Hemodynamics, 105 Human atrium, 28

transmembrane action potential, 340

ventricles, 28 Hypertrophic heart, 28 Hypoxia, 193

Implantable pacemaker, 65 Incidence in Ceylon, 41 Infundibular pressure differences, 218

Intimal hyperplasia, 351 Isoproterenol, 211

Junctional arrhythmias, 156

Kasippu, 41

Labile hypertension, 105 Lactate, 193

Lactatedehydrogenase isoenzymes, 28 Left stellate ganglion stimulation, 218

- $\quad$ ventricular hypertrophy, 149

Limb anomaly, 242

Maintained cardiovascular responses, 164 Maximal walking distance, 114 Mechanical oscillography, 114

Paired stimulation, 211 Papillary muscle, 211 Peripheral circulation, 114

- $\quad$ resistance, 94

Phenoxybenzamine, 237

Phonocardiography, 333

Physical training, 114

Preoperative digitalization, 34

Prevention of atrial fibrillation, 34

Propranolol, 249

Pulmonary artery pressure, 361

blood temperature, 136 
flow, 218

vascular markings, 281

wedge pressure, 361

Quinidine, 249

Rabbit, 94

Rat heart, 28

Red cell electrolytes, 340

Respiratory phase, 281

Right atrial pressure, 361

- $\quad$ bundle branch block, 149

Serum digoxin, 340

Sex differences in cardiac measurements,

281 Siblings, 242 Sick sinus syndrome, 310 Simulated diving, 257 Sleep, 79

Standardization, 129 Steady state, 136

376

Subject Index

Stimulation parameters, 164 'Strokes' in the young adult, 272 Supine exercise, 136

Tricuspid insufficiency, 136 - stenosis, 55

Ultrastructure, 84

Thermal dilution, 94

Thermodilution, 136

Thiamine deficiency, 41

Third heart sound, 333

Toddy, 41

Toxopiasma gondiî, 272

Treatment of tachyarrhythmias, 310

Variability, heart rate, 79

Vasa vasorum, 351

Vasoconstriction, 257

Venous occlusion plethysmography, 114

Ventriculophasic sinus arrhythmia, 65

Whole-body freezing, 237 